\title{
Multicenter randomized phase II trial of atezolizumab with or without cobimetinib in biliary tract cancers
}

\author{
Mark Yarchoan, ${ }^{1}$ Leslie Cope, ${ }^{1}$ Amanda N. Ruggieri, ${ }^{2}$ Robert A. Anders, ${ }^{1}$ Anne M. Noonan, ${ }^{3}$ Laura W. Goff, ${ }^{4}$ Lipika Goyal, ${ }^{5}$ Jill Lacy, ${ }^{6}$ \\ Daneng Li, ${ }^{7}$ Anuj K. Patel, ${ }^{8}$ Aiwu R. He, ${ }^{9}$ Ghassan K. Abou-Alfa, ${ }^{10,11}$ Kristen Spencer, ${ }^{12}$ Edward J. Kim, ${ }^{13}$ S. Lindsey Davis, ${ }^{14}$ \\ Autumn J. McRee, ${ }^{15}$ Paul R. Kunk, ${ }^{16}$ Subir Goyal, ${ }^{2}$ Yuan Liu, ${ }^{2}$ Lauren Dennison, ${ }^{1}$ Stephanie Xavier, ${ }^{1}$ Aditya A. Mohan, ${ }^{1}$ \\ Qingfeng Zhu, ${ }^{1}$ Andrea Wang-Gillam, ${ }^{17}$ Andrew Poklepovic, ${ }^{18}$ Helen X. Chen, ${ }^{19}$ Elad Sharon, ${ }^{19}$ Gregory B. Lesinski, ${ }^{2}$ \\ and Nilofer S. Azad'
}

Johns Hopkins University, Baltimore, Maryland, USA. ${ }^{2}$ Winship Cancer Institute of Emory University, Atlanta, Georgia, USA. ${ }^{3}$ The Ohio State University, Columbus, Ohio, USA. ${ }^{4}$ Vanderbilt-Ingram Cancer Center, Nashville, Tennessee, USA. ${ }^{5}$ Massachusetts Ceneral Hospital Cancer Center, Boston, Massachusetts, USA. ${ }^{6}$ Yale Cancer Center, New Haven, Connecticut, USA. ' ${ }^{7}$ City of Hope, Duarte, California, USA. ${ }^{8}$ Dana-Farber Cancer Institute, Boston, Massachusetts, USA. ${ }^{9}$ Ceorgetown University, Washington, DC, USA. ${ }^{10}$ Memorial Sloan Kettering Cancer Center, New York City, New York, USA. "Weill Medical College at Cornell University, New York City, New York, USA. ${ }^{2}$ Rutgers Cancer Institute, New Brunswick, New Jersey, USA. ${ }^{13}$ UC Davis, Sacramento, California, USA. ${ }^{14}$ University of Colorado Hospital, Aurora, Colorado, USA. ${ }^{15}$ University of North Carolina, Chapel Hill, North Carolina, USA. ${ }^{16}$ University of Virginia, Charlottesville, Virginia, USA. ${ }^{17}$ Washington University in St. Louis, Siteman Cancer Center, St. Louis, Missouri, USA. ${ }^{18}$ Virginia Commonwealth University, Massey Cancer Center, Richmond, Virginia, USA. ${ }^{19} \mathrm{NCI}$ Cancer Therapy Evaluation Program, Bethesda, Maryland, USA.

BACKCROUND. MEK inhibitors have limited activity in biliary tract cancers (BTCs) as monotherapy but are hypothesized to enhance responses to programmed death ligand 1 (PD-L1) inhibition.

METHODS. This open-label phase II study randomized patients with BTC to atezolizumab (anti-PD-L1) as monotherapy or in combination with cobimetinib (MEK inhibitor). Eligible patients had unresectable BTC with 1 to 2 lines of prior therapy in the metastatic setting, measurable disease, and Eastern Cooperative Oncology Group (ECOC) performance status less than or equal to 1 . The primary endpoint was progression-free survival (PFS).

RESULTS. Seventy-seven patients were randomized and received study therapy. The trial met its primary endpoint, with a median PFS of 3.65 months in the combination arm versus 1.87 months in the monotherapy arm (HR $0.58,90 \%$ $\mathrm{Cl} 0.35-0.93$, 1-tail $P=0.027$ ). One patient in the combination arm (3.3\%) and 1 patient in the monotherapy arm (2.8\%) had a partial response. Combination therapy was associated with more rash, gastrointestinal events, CPK elevations, and thrombocytopenia. Exploratory analysis of tumor biopsies revealed enhanced expression of antigen processing and presentation genes and an increase in CD8/FoxP3 ratios with combination treatment. Patients with higher baseline or lower fold changes in expression of certain inhibitory ligands (LAC3, BTLA, VISTA) on circulating T cells had evidence of greater clinical benefit from the combination.

CONCLUSION. The combination of atezolizumab plus cobimetinib prolonged PFS as compared with atezolizumab monotherapy, but the low response rate in both arms highlights the immune-resistant nature of BTCs.

TRIAL REGISTRATION. ClinicalTrials.gov NCT03201458.

FUNDING. National Cancer Institute (NCI) Experimental Therapeutics Clinical Trials Network (ETCTN); F. HoffmannLa Roche, Ltd.; NCI, NIH (R01 CA228414-01 and UM1CA186691); NCl's Specialized Program of Research Excellence (SPORE) in Gastrointestinal Cancers (P50 CA062924); NIH Center Core Grant (P30 CA006973); and the Passano Foundation.

Conflict of interest: MY received grant and research support from Incyte, BristolMyers Squibb, Exelixis, and Genentech/Roche and acted in a consulting or advisory role for Eisai, Exelixis, AstraZeneca, Genentech/Roche, and Geneos. NSA acted in a consulting or advisory role for QED, AstraZeneca, Mirati, and Taiho; received research funding from Celgene, Genentech, Astex Pharmaceuticals, Agios, Merck, BristolMyers Squibb, Syndax, Array BioPharma, Intensity Therapeutics, Atlas, Taiho, LOXO and EMD Serono. CBL has consulted for and received compensation from ProDa Biotech, LLC and has received research funding from Merck and Co., Bristol-Myers Squibb, Boerhinger-Ingelheim, and Vaccinex.

Copyright: @ 2021, American Society for Clinical Investigation

Submitted: June 25, 2021; Accepted: October 19, 2021; Published: December 15, 2021 Reference information: / Clin Invest. 2021;131(24):e152670

https://doi.org/10.1172/JCl152670.

\section{Introduction}

Biliary tract cancers (BTCs) are cancers arising from the biliary epithelial cells and are historically subcategorized anatomically as intrahepatic cholangiocarcinoma, extrahepatic cholangiocarcinoma, and gallbladder cancer. The incidence of BTCs is increasing, paralleling global trends in etiologic risk factors such as obesity and the metabolic syndrome, as well as improved awareness and diagnostic expertise (1-3). Most patients have an advanced stage at diagnosis, and outcomes are generally poor. Patients receiving systemic therapy including the standard frontline therapy for unresectable BTC with gemcitabine plus cisplatin have 


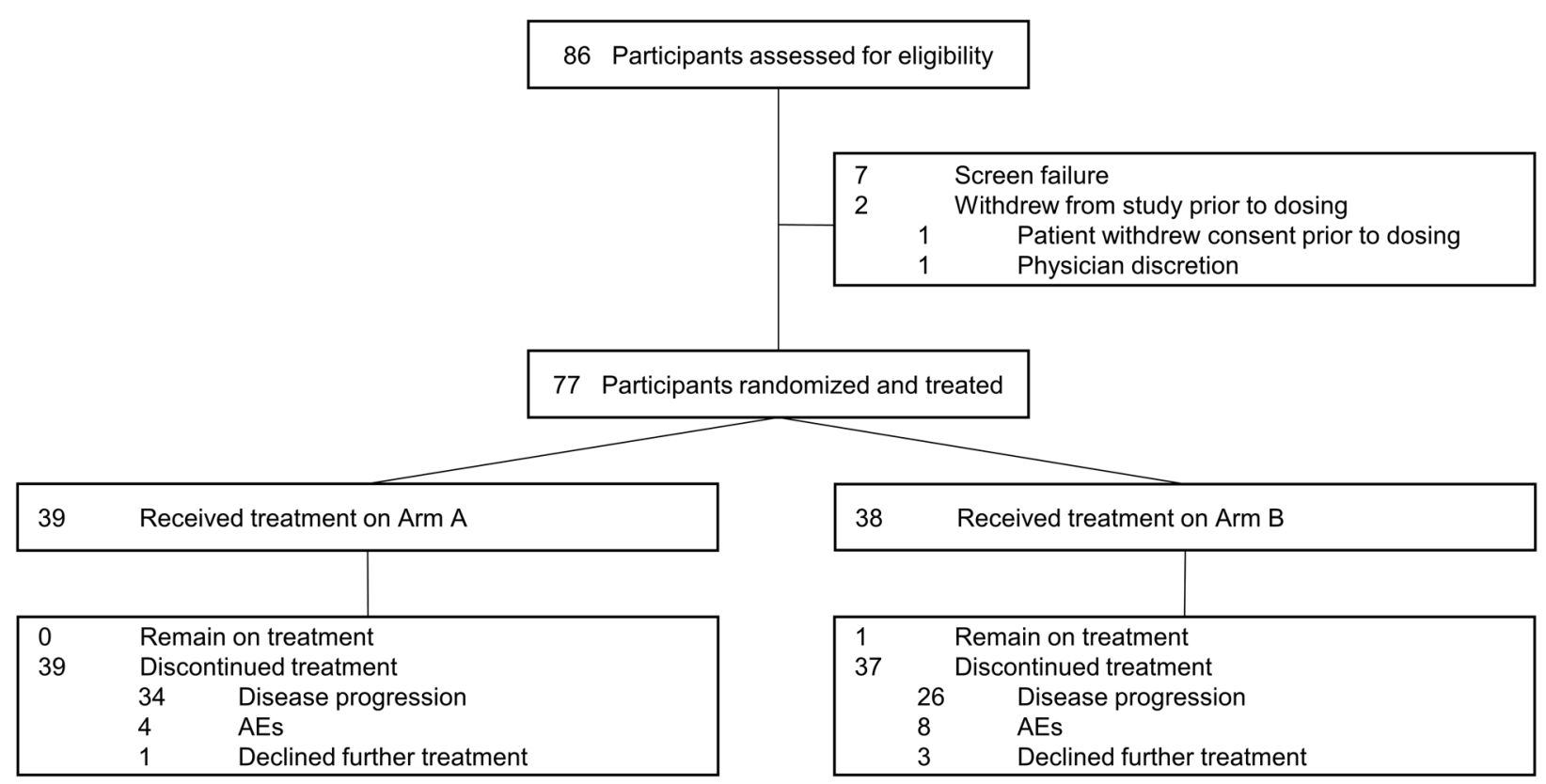

Figure 1. Consort diagram.

a modest median overall survival (OS) of less than 1 year (4). A subset of patients with BTC have potentially actionable molecular alterations such as FGFR2 fusions or rearrangements or IDH1 mutations and may benefit from treatment with molecularly targeted therapies $(5,6)$. The benefit of chemotherapy in the second line setting is limited (7-10), underscoring the need for additional treatment options for these patients.

The mitogen-activated protein kinase (MAPK) cascade is a critical pathway for cell proliferation, and dysregulation of this pathway is a hallmark of BTC (11-14). Mitogen/extracellular signal-regulated kinase (MEK) is a key intermediary component of the MAPK pathway, and is an attractive target in principle for therapeutic intervention in BTCs (15). MEK inhibitors have thus far demonstrated some limited single agent activity in unselected BTCs (16-19). Similarly, immune checkpoint inhibitors (ICIs) targeting programmed cell death protein 1 (PD-1) or its ligand, PD-L1, have some limited activity in BTCs as monotherapy, with response rates of approximately $6 \%$ to $11 \%$ in the largest reported prospective clinical trials, all single-arm studies (20-23). The development of novel therapeutic combinations that can extend the clinical benefit of ICIs to immunologically resistant tumors such as BTCs remains a significant challenge.

MEK inhibitors have shown immunomodulatory effects and substantial efficacy when combined with PD-1 and PD-L1 inhibitors in preclinical models of colon cancer, breast cancer, and melanoma (24-26). Targeting of the MAPK pathway through MEK inhibition is hypothesized to modulate the tumor immune microenvironment (TME) through effects on tumor cells and direct effects on immune cells, resulting in enhanced major histocompatibility complex class I (MHC-1) expression, PD-L1 expression, and $\mathrm{CD}^{+} \mathrm{T}$ cell infiltration $(25,27-32)$. Although acceptable safety and promising clinical activity was observed in initial phase I studies combining a MEK inhibitor plus PD-L1 inhibition with notable clinical responses in colorectal cancer, this treatment combina- tion recently failed to show compelling immune activity in a confirmatory phase III clinical study in colorectal cancer (33). Here we report the results of a multicenter randomized phase II trial of atezolizumab, an inhibitor of PD-L1, as monotherapy or in combination with the MEK inhibitor cobimetinib in BTCs.

\section{Results}

Patients and treatment. From February 2018, to October 2018, 86 participants were assessed for eligibility, of whom 77 were randomized and received treatment with atezolizumab monotherapy $(n=39)$ or atezolizumab plus cobimetinib $(n=38)$ at the NCI's Experimental Therapeutics Clinical Trials Network (ETCTN) sites in the United States (Figure 1). Baseline demographic and disease characteristics were similar between the 2 groups of randomized patients and are shown in Table 1 . In total, 43 patients (55.8\%) had intrahepatic cholangiocarcinoma, 15 patients (19.5\%) had extrahepatic cholangiocarcinoma, and 19 patients (24.7\%) had gallbladder cancer. Most patients (61.0\%) had 1 prior regimen in the metastatic setting, whereas $39.0 \%$ had 2 prior systemic regimens in the metastatic setting. One participant in each study arm had known mismatch repair deficiency (MMRd), and no other patients had a known tumor mutation burden (TMB) of greater than 10 mutations/Mb.

Clinical activity. The study met its primary endpoint, demonstrating a significantly longer PFS for patients in the combination treatment than for those in the single treatment group (HR 0.58, $90 \%$ CI $0.35-0.93), P=0.027$ by a 1 -sided, stratified log rank test). An unstratified Kaplan-Meier plot is shown in Figure 2, and the 4-, 6-, and 12-month PFS for each study arm are shown in Supplemental Table 1. The median PFS for single and combination therapies was 1.87 months and 3.65 months, respectively. The 4-month PFS rate for the combination and monotherapy arms were $44.6 \%$ and 9.4\%, respectively. The 6-month PFS rates were $22.3 \%$ and $9.4 \%$, and the 12-month PFS rates were 13.4 and $0 \%$, for the combination 


\section{Table 1. Baseline demographic and clinical characteristics of the study patients}

\begin{tabular}{|c|c|c|}
\hline & Single, $n=39$ & Combination, $n=38$ \\
\hline \multicolumn{3}{|l|}{ Tumor type } \\
\hline Extrahepatic cholangiocarcinoma (EHC) & 7 & 8 \\
\hline Intrahepatic cholangiocarcinoma (IHC) & 21 & 22 \\
\hline Gallbladder cancer (GBC) & 11 & 8 \\
\hline \multicolumn{3}{|l|}{ Age, years } \\
\hline$<50$ & 1 & 4 \\
\hline 50 to 70 & 28 & 24 \\
\hline$>70$ & 10 & 10 \\
\hline \multicolumn{3}{|l|}{ Sex } \\
\hline Female & 27 & 21 \\
\hline Male & 12 & 17 \\
\hline \multicolumn{3}{|l|}{ ECOC performance status } \\
\hline 0 & 16 & 8 \\
\hline 1 & 23 & 30 \\
\hline \multicolumn{3}{|l|}{ Race and ethnicity } \\
\hline White, not Hispanic & 28 & 27 \\
\hline Hispanic & 4 & 7 \\
\hline Black & 5 & 2 \\
\hline Asian & 2 & 1 \\
\hline Not reported & 0 & 1 \\
\hline \multicolumn{3}{|l|}{ Prior therapies } \\
\hline 1 & 23 & 24 \\
\hline 2 & 16 & 14 \\
\hline \multicolumn{3}{|l|}{ MSI-H/MMRd } \\
\hline Positive & 1 & 1 \\
\hline Negative or unknown & 38 & 37 \\
\hline \multicolumn{3}{|l|}{ Mutations } \\
\hline Known KRAS mutation & 7 & 8 \\
\hline Known IDH1 mutation & 1 & 3 \\
\hline Known FGFR2 mutation & 2 & 4 \\
\hline Known TP53 mutation & 5 & 6 \\
\hline Known CDKN2A/B mutation & 3 & 5 \\
\hline \multicolumn{3}{|l|}{ CA19-9 } \\
\hline Elevated (>37 U/mL) & 24 & 26 \\
\hline Not elevated & 13 & 10 \\
\hline Unknown & 2 & 2 \\
\hline
\end{tabular}

and monotherapy arms, respectively. In an unplanned, post hoc analysis, there was a suggestion that the benefit observed for combination therapy was specific to intrahepatic cholangiocarcinoma. This group of patients achieved a median PFS of 4.44 months on combination therapy, whereas all other groups had median PFS of 1.71 months to 2.07 months regardless of treatment intervention. A Kaplan-Meier plot stratified by primary disease site is shown in Supplemental Figure 1.

In total, 36 patients in the monotherapy arm of the study and 30 patients in the combination arm of the study were evaluable for response. The evaluable population included patients removed from study prior to the first radiographic evaluation time point for clinical progression or death from tumor progression. Among evaluable patients, 1 patient (2.8\%) had an objective response in the monotherapy arm and 1 patient (3.3\%) had an objective response in the combination arm. Disease control as demonstrated by par- tial response plus stable disease was seen in $46.7 \%$ versus $30.6 \%$ of patients treated with cobimetinib plus atezolizumab versus atezolizumab alone, respectively; this difference was not statistically significant $(P=0.21)$. Response Evaluation Criteria in Solid Tumors version 1.1 (RECIST 1.1) responses for all subjects who had RECIST 1.1 evaluable scans are shown in Figure 3, and objective responses for all evaluable patients are summarized in Table 2.

The 2 responders in our study included a patient with gallbladder cancer treated with atezolizumab monotherapy and a patient with intrahepatic cholangiocarcinoma treated with combination therapy. These responses were both durable; the monotherapy patient response lasted approximately 10 months and the combination therapy response is ongoing more than 2 years after starting therapy. No molecular information was available for the responder with gallbladder cancer, whereas the patient with intrahepatic cholangiocarcinoma had known FGFR2, PIK3CA, and TP53 mutations. One patient per treatment arm had known MMRd, of whom neither responded to study therapy. The patient with MMRd receiving monotherapy had progressive disease as a best response to therapy, whereas the patient with MMRd randomized to the combination treatment arm was removed for intolerance prior to the first tumor evaluation.

Fifty patients (64.9\%) had an elevated CA19-9 at study baseline, and CA19-9 levels were followed routinely for this subset of patients. A waterfall plot of maximum CA19-9 decline on study therapy for all subjects with an elevated CA19-9 at baseline is shown in Supplemental Figure 2. Among patients with an elevated CA19-9 at baseline, a decrease of $30 \%$ or more was observed in 4 of 24 patients $(16.7 \%)$ in monotherapy arm and 7 of 26 patients (26.9\%) in the combination arm.

At the time of final analysis, 68 of 77 subjects had died. OS was not different between the groups, though the study was not powered for this endpoint ( $P=0.410$ by 1 -sided, log-rank test, stratified by tumor site). As shown in Supplemental Figure 3, median survival by treatment group and primary tumor location strata varied widely (95-234 days).

Safety. All patients in either treatment arm who received at least 1 dose of study drug were included in the safety analysis. Treatment-related adverse events (TRAEs) that were reported in more than $10 \%$ of patients in either treatment arm, as well as all treatment related grade 3 or higher events, are shown in Table 3. The combination of cobimetinib and atezolizumab was associated with a higher frequency of some adverse events than atezolizumab monotherapy, including rash, pruritus, dry mouth, diarrhea, nausea or vomiting, thrombocytopenia, and CPK elevations. Most of these events were grade 1 to 2 and expected toxicities of cobimetinib.

TRAEs of any grade occurred comparably in both arms, affecting 33 subjects $(84.6 \%)$ in the monotherapy arm and 33 subjects $(86.8 \%)$ in the combination treatment arm. Similarly, treatment-related grade 3 events occurred in 15 patients $(38.5 \%)$ in the monotherapy arm and 17 patients $(44.7 \%)$ in the combination arm. There were no treatment-related grade 4 or grade 5 events in either treatment $\mathrm{arm}$. There were 13 deaths on study that were all determined to be unrelated to study treatment. Most were related to disease progression $(n=12)$ or to sepsis in the setting of disease progression $(n=1)$.

Dose interruptions due to AEs occurred in 3 patients (7.9\%) in the combination treatment arm and 4 patients (10.3\%) in the 

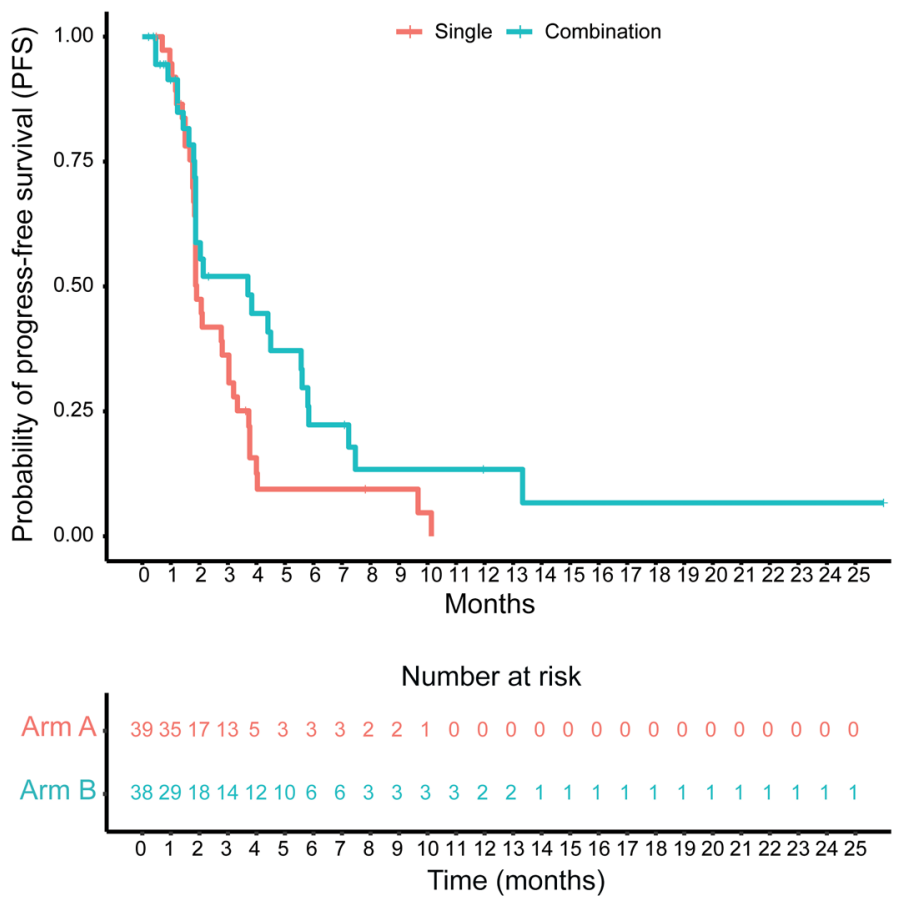

Figure 2. Unstratified Kaplan-Meier plot of PFS for atezolizumab monotherapy (Arm A) and atezolizumab plus cobimetinib (Arm B).

monotherapy arm. Dose reductions of cobimetinib occurred in 6 patients $(15.8 \%)$ in the combination arm. A total of 4 patients (10.3\%) receiving atezolizumab monotherapy and 8 patients (21.1\%) receiving combination therapy with cobimetinib plus atezolizumab discontinued therapy due to AEs. Although there were twice as many discontinuations in the combination arm as the monotherapy arm due to AEs, neither inverse KaplanMeier analysis nor Fisher's exact test for study discontinuation rate due to adverse events was significant $(P=0.22)$. Although most patients who discontinued combination therapy due to AEs had multiple drug-related AEs that may have prompted discontinuation, nausea, vomiting, and diarrhea were the most common AEs reported by these patients.

Biomarker analysis. Biopsies were mandated when feasible for all subjects at baseline and at approximately day 21 of study therapy. Nine patients in each treatment arm had paired pretreatment and on-treatment biopsies, although tumor tissue was limited on some of these specimens. The randomized nature of the study enabled us to interrogate the additive effects of MEK inhibition with cobimetinib on the tumor immune microenvironment by immunohistochemistry (IHC) in a subset of patients. Prior human serial biopsy studies have reported that MEK inhibition can increase $\mathrm{CD} 8^{+} \mathrm{T}$ cell infiltration and antigen expression. However, $\mathrm{CD} 8^{+} \mathrm{T}$ cell and human leucocyte antigen (HLA) expression were highly variable across the samples analyzed. PD-L1 expression was negative in the majority of samples and did not increase with therapy on either treatment arm. We did observe an increase in the CD8 ${ }^{+}$cytotoxic $\mathrm{T}$ cells to FoxP3 $\mathrm{T}$ regulatory cell ratio in the combination therapy arm relative to the single treatment arm (Supplemental Figure 4).

We also explored differences between the combination and monotherapy treatment arms by RNA at day 21 using the nCounter
Nanostring PanCancer Immune Profiling Panel (Figure 4). As compared with atezolizumab monotherapy, there was a trend toward higher expression of antigen-processing and presentation genes, including TAP-associated glycoprotein (TAPBP), immunoproteasome expression (proteasome subunit beta type-8, PSMB8), and human leukocyte antigen (HLA) in the combination arm, as well as enhanced expression of interferon signaling pathway member interferon-induced transmembrane protein 2 (IFITM2). While these differences are exploratory in nature, they support the idea that cobimetinib may invigorate antitumor immunity in the TME and are in broad agreement with preclinical models of MEK inhibition $(26,34)$.

Correlative analysis of cryopreserved PBMCs from patients was conducted in an exploratory manner to assess $\mathrm{T}$ cellfocused biomarkers and relationship to clinical outcome measures and treatment. For this analysis, we focused on differences in biomarkers attributable to the combination treatment versus monotherapy by incorporating an interaction effect. These data are summarized in Table 4 . At baseline, patients in the combination arm with a higher than median percentage of LAG3 $^{+} \mathrm{CD}^{+} \mathrm{T}$ cells $(\mathrm{HR}=0.43, P=0.035)$ had better OS than in the monotherapy arm, while more $\mathrm{TIM}^{+} \mathrm{CD}^{+} \mathrm{T}$ cells (OR $=4.8, P=0.033$ ) were indicative of more favorable response by RECIST in the combination arm versus the monotherapy arm. These biomarkers, however, were not significant predictors of better survival or clinical response at baseline when data from all patients, regardless of treatment arm, were compiled for analysis. In addition to assessing biomarkers at baseline, we also evaluated fold-change in cell percentages from baseline to cycle 2 day 1 (C2D1). This approach revealed that patients in the combination arm with a decrease in several $\mathrm{T}$ cell-focused biomarkers had better OS. These included decreases in LAG3 ${ }^{+}(\mathrm{HR}=0.36$, $P=0.024)$ and $\mathrm{BTLA}^{+}(\mathrm{HR}=0.31, P=0.014) \mathrm{CD}^{+} \mathrm{T}$ cells. Complementing these data was the observation that patients in Arm $\mathrm{B}$ with a decrease in $\mathrm{VISTA}^{+} \mathrm{CD}^{+} \mathrm{T}$ cells $(\mathrm{HR}=0.23, P=0.004)$ from baseline to C2D1 had significantly longer PFS. Because our subgroup analysis indicated the benefit of combination therapy might be limited to the intrahepatic subset, we also looked to see if this group had baseline differences in the expression of inhibitory $\mathrm{T}$ cell checkpoints that were associated with evidence of greater clinical benefit from the combination across the entire study population. However, we found no difference in the expression of LAG3 or TIM3 on T cells at baseline in this subgroup.

\section{Discussion}

This multicenter, open-label, randomized phase II trial of patients with advanced BTC met its primary endpoint, showing an improvement in PFS when cobimetinib was added to atezolizum$a b$ as compared with atezolizumab monotherapy. These data need to be interpreted in the context of a rapidly evolving treatment landscape, with recent reports of 5-FU plus oxaliplatin (9) or 5-FU plus liposomal irinotecan (35) as second line treatment options for unselected BTC, and the development of multiple targeted therapies for the subsets of patients with potentially actionable molecular alterations $(5,6,36,37)$. The safety of the atezolizumab plus cobimetinib combination was consistent with the toxicity profile of the 2 individual drugs and with the prior experience of this 
A

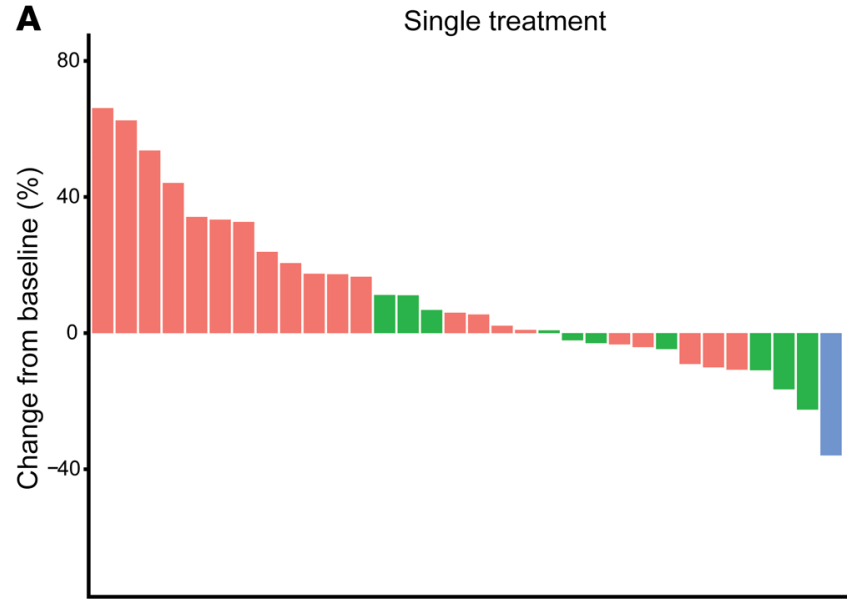

B

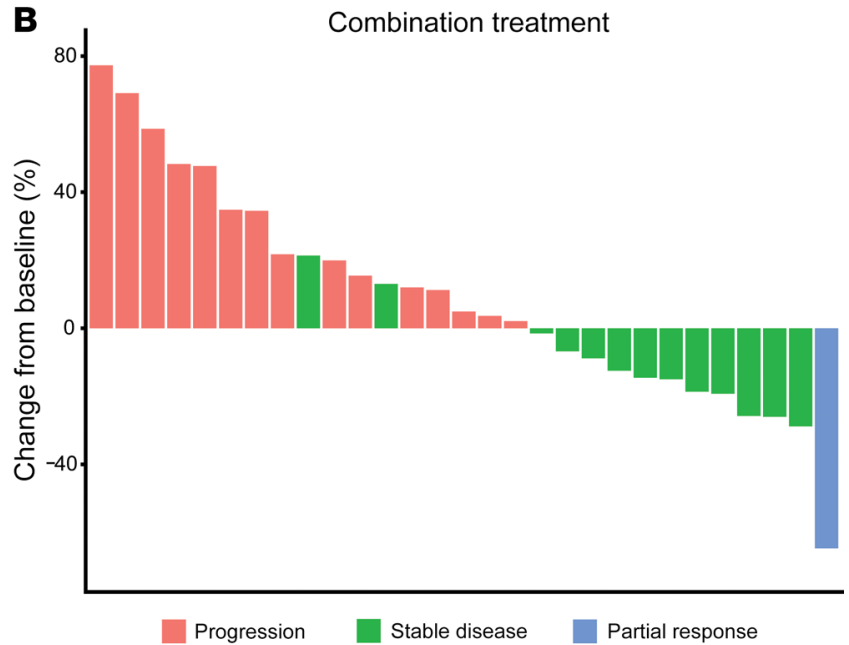

combination in other tumor types $(32,33)$. Although some treatment-related adverse events were more common in the combination treatment arm, the study drugs predominantly had nonoverlapping adverse event profiles and were tolerable in combination.

This study is, to our knowledge, the largest randomized study of immune checkpoint-based therapy for patients with BTC reported to date, and builds upon other studies demonstrating the limited clinical activity of ICIs as monotherapy in this disease (20-23). Similarly, although MAPK pathway activation is common in BTC, MEK inhibitors have shown only modest activity as monotherapy $(16-19,38)$ or in combination with chemotherapy (19). This study was conducted because of preclinical research indicating the potential for synergy between MEK inhibition and systemic immunotherapies $(25,27-32)$. While our study did provide evidence that MEK inhibitors may have some benefit in the context of systemic immunotherapy for BTCs, whether the modest PFS benefit observed in our study reflects additive effects of the individual therapies or true synergy resulting from MEK immunomodulation of the tumor microenvironment is unclear.

We did not observe an absolute increase in $\mathrm{CD} 8^{+} \mathrm{T}$ cell infiltration with combination therapy, as reported previously in a study of cobimetinib monotherapy (32), but we did observe an increase in $\mathrm{CD}^{+} \mathrm{T}$ cells to $\mathrm{FoxP}^{+} \mathrm{T}$ regulatory cell ratio in the combination arm as compared with the monotherapy arm. The ratio of $\mathrm{CD}^{+} /$
Figure 3. Best response by RECIST 1. 1 among the evaluable patients treated with atezolizumab monotherapy and atezolizumab plus cobimetinib. (A) Monotherapy. (B) Combination therapy.

FoxP3 $^{+}$Tregs within tumors are hypothesized to be prognostic as well as predictive of responses to immunotherapy in a variety of cancer settings (39-41). Our data provide initial evidence that MEK inhibitors are immunomodulatory in BTC and augment antitumor $\mathrm{T}$ cell immunity and/or inhibit immunosuppressive axes within the tumor microenvironment. The observation of enhanced expression of multiple genes involved antigen-processing and presentation in the present study is also consistent with preclinical studies $(26,34)$. We also observed that patients with higher baseline expression or smaller fold changes in the expression of multiple inhibitory ligands (LAG3, BTLA, VISTA) on circulating $\mathrm{T}$ cells had evidence of greater clinical benefit from the combination. This work is hypothesis generating, but suggests the possibility that the reversal or prevention of $\mathrm{T}$ cell exhaustion is a key mechanism of systemic MEK inhibition immunomodulation, as previously shown in preclinical models $(26,34)$. Overall, these clinical trial biomarkers suggest that MEK inhibitor therapy modulated the response to anti-PD-L1 immunotherapy and was of benefit for some patients. The small number of adequate biopsies available for correlative analysis, and incomplete molecular information for the majority of patients, were significant limitations of the present study.

The low response rate observed in the combination arm of the study indicates that the observed changes to the TME were insufficient to cause tumor regression in the vast majority of patients and highlights the immune-resistant nature of BTC. While MEK inhibitors may augment antigen presentation and protect against $\mathrm{T}$ cell exhaustion, they may also impair $\mathrm{T}$ cell priming and activation, which may limit the efficacy of this combination and provides a rationale for combining these agents with a $\mathrm{T}$ cell agonist (26, 34, 42-44). Preclinical models support the efficacy of MEK inhibitors plus PD-L1 inhibitors plus T cell costimulatory agents (42-44). The hypothesis that the addition of a costimulatory agent can restore $\mathrm{T}$ cell function when added to MEK inhibition plus PD-L1 inhibitors is being explored in a follow-on randomized study of cobimetinib, atezolizumab, and the CD27 T cell agonist varlilumab in BTCs, conducted through our multi-institutional

Table 2. Objective response summary for evaluable patients

\begin{tabular}{lcc} 
Response, $n(\%)$ & Single, $\boldsymbol{n}=\mathbf{3 6}$ & Combination, $\boldsymbol{n}=\mathbf{3 0}$ \\
\hline Complete response (CR) & 0 & 0 \\
Partial response (PR) & $1(2.8)$ & $1(3.3)$ \\
\hline Stable disease (SD) & $10(27.8)$ & $13(43.3)$ \\
$\quad$ Progressive disease (PD) & $25(69.4)$ & $16(53.3)$ \\
Rate of objective response (CR+PR) & & \\
$\quad n(\%)$ & $1(2.8)$ & $1(3.3)$ \\
Rate of disease control (CR+PR+SD) & & $14(46.7)$ \\
$\quad n(\%)$ & $11(30.6)$ & \\
\hline
\end{tabular}


Table 3. Treatment-related AEs



Treatment-related AEs occurring in more than $10 \%$ of patients in either treatment arm, and all treatment-related grade 3 events. There were no treatmentrelated grade 4 or grade 5 events in either treatment arm. Rash, pruritus, dry mouth, diarrhea, nausea or vomiting, thrombocytopenia, and CPK elevations were more common in the combination treatment arm (cobimetinib plus atezolizumab).

collaborative group within the NCI's ETCTN (ClinicalTrials.gov identifier: NCT04941287).

In summary, this randomized phase II study met its primary endpoint, indicating that the combination of cobimetinib and atezolizumab may improve PFS and has a manageable safety profile. However, objective response rates were low in both treatment groups, and we did not observe an increase in OS with the combination group. Due to the open-label nature of the study, we cannot exclude the possibility that the benefit in PFS with combination therapy was due to biases in assessment of progression rather than differences in clinical activity between the study groups. Our finding that the benefit of combination therapy was specific to intra- 


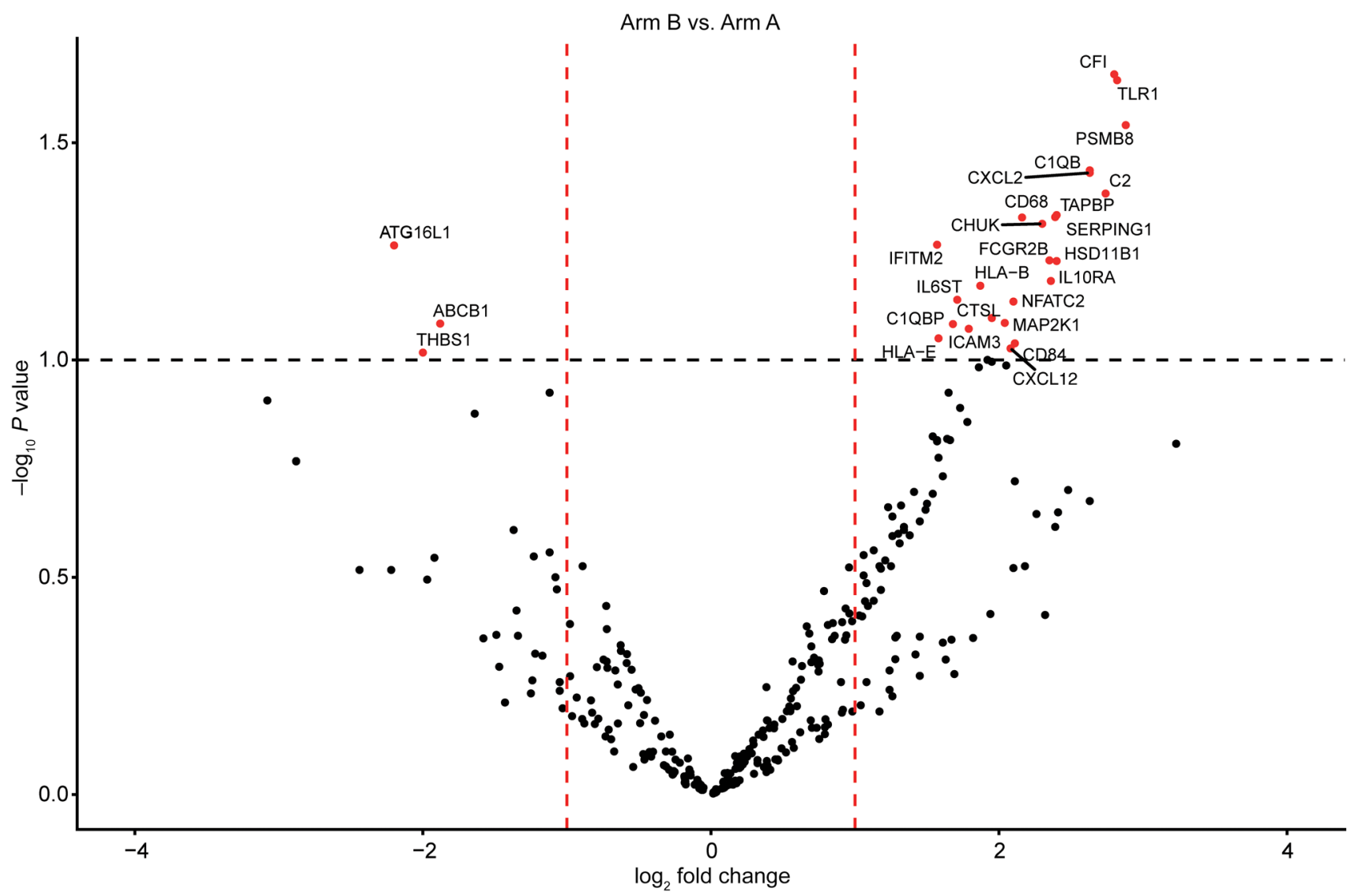

Figure 4. Exploratory gene expression profiling. Tumor biopsies obtained at approximately treatment day 21 revealed differences in the expression of multiple genes in the combination treatment arm versus the monotherapy arm. Enhanced expression of genes involved in antigen processing and presentation (including TAP-associated glycoprotein [TAPBP], proteasome subunit beta type-8 [PSMB8], and human leukocyte antigen [HLA]) as well as an increase in interferon signaling pathway member interferon-induced transmembrane protein 2 (IFITM2) in the combination arm, are consistent with preclinical models of MEK inhibition. Quality control assessment and data normalization were performed using the default settings for positive controls and the housekeeping genes in nSolver Analysis Software (NanoString Technologies) without $P$ value adjustment, $n=6$ samples per group.

hepatic cholangiocarcinoma in an unplanned post hoc analysis is hypothesis generating and warrants further investigation. Intrahepatic cholangiocarcinoma has unique molecular altercations not found in other anatomically defined subsets of BTC (45), and may respond distinctly to MEK inhibition. Immunotherapy combinations including cobimetinib plus atezolizumab warrant additional investigation in BTC, potentially as a basis for added novel combinatorial immunotherapy approaches.

\section{Methods}

Patients and eligibility criteria. At the time that the study was initiated, no treatment had ever conclusively shown benefit in second line treatment of cholangiocarcinoma (7) and treatment guidelines did not provide a recommendation for second line therapy. Eligible patients had received at least 1 and not more than 2 prior lines of systemic therapy in the metastatic setting. In addition, eligible patients were at least 18 years of age, had an ECOG performance status score of 0 or 1 , and had pathologically confirmed intrahepatic cholangiocarcinoma, extrahepatic cholangiocarcinoma, or gallbladder carcinoma. Patients with ampullary carcinoma were excluded. Eligible patients also had measurable disease according to RECIST 1.1 and had adequate hematologic, hepatic, renal, and cardiac function. Patients with prior treat- ment with a PD-1 or PD-L1 inhibitor or MEK inhibitor, a history of autoimmune disease or treatment with systemic immunosuppressive medications, or uncontrolled intercurrent illness were excluded. Due to the potential for drug interactions with cobimetinib, patients receiving any medications or substances that are strong inhibitors or inducers of CYP3A4 enzymes were also ineligible.

Randomization and treatment. Patients were randomly assigned in a 1:1 ratio to receive atezolizumab $840 \mathrm{mg}$ i.v. every 2 weeks (Arm A), or cobimetinib $60 \mathrm{mg}$ daily (21 days on/7 days off) plus atezolizumab 840 mg i.v. every 2 weeks (Arm B). Randomization was stratified after the screening phase according to the site of disease: (a) gallbladder cancer; (b) intrahepatic; and (c) extrahepatic cholangiocarcinoma. Randomization was performed centrally and implemented through a web-based response system (Theradex Interactive Web Response System). For reporting of race and ethnicity, all classifications were made by the investigators, and demographic options were defined by the investigators.

The study treatment was continued until patients withdrew consent, developed unacceptable adverse effects, were not candidates for further treatment in the judgment of the investigator, or developed progression not meeting criteria for continuation past progression. Treatment past progression was allowed for patients on both treat- 


\section{Table 4. Biomarker correlative analyses in subgroups defined by biomarkers for OS, PFS, and best response}

\begin{tabular}{|c|c|c|c|}
\hline Clinical endpoint & Biomarker & Hazard ratio & $P$ value \\
\hline Best response ${ }^{A}$ & $\mathrm{CD}^{+} \mathrm{TIM}^{+}$above the median at baseline & $4.80(1.14-20.27)$ & 0.033 \\
\hline \multirow[t]{3}{*}{$O S^{B}$} & $\mathrm{CD}^{+} \mathrm{LAC3}^{+}$above median at baseline & $0.43(0.20-0.94)$ & 0.035 \\
\hline & $\mathrm{CD}^{+} \mathrm{LAC3}^{+}$fold-change at $\mathrm{C} 2 \mathrm{D} 1$ below the median & $0.36(0.14-0.87)$ & 0.024 \\
\hline & $\mathrm{CD}^{+} \mathrm{BTLA}^{+}$fold-change at $\mathrm{C} 2 \mathrm{D} 1$ below the median & $0.31(0.12-0.79)$ & 0.014 \\
\hline PFS $^{B}$ & $\mathrm{CD}^{+} \mathrm{VISTA}^{+}$fold-change at $\mathrm{C} 2 \mathrm{D} 1$ below the median & $0.23(0.08-0.63)$ & 0.004 \\
\hline
\end{tabular}

ALogistic regression with estimated odds ratio and $95 \% \mathrm{Cl}$ for comparison between Arm B and Arm A. ${ }^{B}$ Cox proportional hazard mode with estimated hazard ratio and $95 \% \mathrm{Cl}$ for comparison between Arm B and Arm A.

ment arms, but only in cases of clinical benefit and absence of signs and symptoms of unequivocal progression, as assessed by the investigator. Treatment discontinuation was mandated on radiographic progression at any subsequent evaluation. No dose reductions were permitted for atezolizumab, but cobimetinib dose reductions were permitted in $20 \mathrm{mg}$ increments for adverse events (i.e., grade $\geq 2$; see the study protocol). Patients experiencing intolerance to 1 of the 2 therapies in the combination study arm were permitted to continue cobimetinib or atezolizumab monotherapy.

Study endpoints. The primary objective of the trial was to determine whether the combination of cobimetinib and atezolizumab yields clinically compelling antitumor activity measured as PFS. The primary endpoint of PFS was defined as the duration of time from date of randomization to time of progression or death. PFS was assessed by the treating investigator according to RECIST 1.1. Tumor assessments were obtained at baseline and approximately every 8 weeks while on study regardless of treatment arm. The evaluable population for the primary study endpoint included all subjects who had completed at least 1 dose of therapy and had at least 1 follow-up scan or came off of study treatment for clinical progression prior to the first follow-up scan (i.e., clinical progression). Patients removed from study for clinical or radiographic progression prior to the first restaging scan were considered to have had progressive disease as a best response to study therapy for objective response rate. The analysis was stratified by the site of disease as described above. Additional secondary endpoints included safety, objective response rate (ORR, assessed by RECIST 1.1) and OS.

Immune profiling of tumor and blood biospecimens. Patients with tumor that was amenable to biopsy underwent core biopsies at study baseline and at cycle 1 day 21 (+/- 5 days). The biopsies were paraffin embedded and used to assess the effect of treatment arm on changes in the tumor immune microenvironment. Staining of multiple immune markers by IHC was conducted in a Central Reference Clinical Laboratory Improvement Amendments Laboratory, and analysis of immune cell populations was performed as previously described (46). Analysis of PD-L1 and HLA expression was performed manually by a single hepatobiliary pathologist who was blinded to treatment arm and clinical outcomes. RNA from patient biopsies were extracted using Qiagen AllPrep DNA/RNA. Large pieces of tissue were first ground using Thermo Fisher Scientific $15 \mathrm{~mL}$ closed tissue grinder (catalog 02-542-09). RLT buffer was added and the lysate was pipetted into a Qiashredder spin column (Qiagen, catalog 79656). The lysate was transferred to an AllPrep RNA column (Qiagen) and purified following the manufacturer's All-
Prep RNA protocol. RNA was analyzed both on the Nanodrop 2000 and the Agilent 2100 Bioanalyzer. RNA was run on the NanoString Human Pan Cancer Immune Profiling Panel. Samples were analyzed using Nanostring nSolver 4.0 software. All RNA analyses were exploratory in nature, and reported without correction for multiple comparisons.

Peripheral blood was drawn prior to administration of cobimetinib and/or atezolizumab at baseline, cycle 1 day 15, and cycle 2 day 1 . Peripheral blood mononuclear cells (PBMCs) were collected by density gradient centrifugation as described (47). Cryopreserved PBMCs underwent flow cytometric analysis for frequency of $\mathrm{T}$ cell subsets and for expression of ICI molecules. PBMCs were stained with anti-CD45-PerCP, anti-CD3-BV510, anti-CD4-PE/ Cy5, anti-CD8-APC/Cy7, anti-LAG3-AF700, anti-BTLA-PE/Cy7, anti-VISTA-APC, and anti-TIM3-SB600. Antibodies used for PBMC staining are shown in Supplemental Table 2. Immune cell subsets were defined as: $\mathrm{CD}^{+} \mathrm{T}$ cells $\mathrm{CD}^{+} / \mathrm{CD}^{+}, \mathrm{CD}^{+}{ }^{+} \mathrm{T}$ cells $\mathrm{CD}^{+} / \mathrm{CD}^{+}$, Th1 cells $\mathrm{CD}^{+} / \mathrm{CD}^{+} / \mathrm{tBet}^{+}$, Th2 cells $\mathrm{CD}^{+} / \mathrm{CD}^{+} / \mathrm{GATA}^{+}$, and Th17 cells $\mathrm{CD}^{+} / \mathrm{CD}^{+} / \mathrm{ROR} \gamma \mathrm{t}^{+}$. ICI checkpoint molecules were assessed on both $\mathrm{CD}^{+}$and $\mathrm{CD}^{+} \mathrm{T}$ cells. PBMCs were fixed and permeabilized using the eBioscience FoxP3/Transcription Factor Fixation/Permeabilization kit (Invitrogen). Data were acquired using a Cytek Aurora flow cytometer (Cytek Biosciences).

Statistics. With PFS as the primary endpoint, we planned to enroll 82 patients with metastatic cholangiocarcinoma, with the goal of accruing at least 76 evaluable subjects. PFS within each treatment arm was summarized using Kaplan-Meier plots, and compared between groups, under the assumption of Cox proportional hazards, using the stratified log-rank test to account for tumor site. We estimated that this design yielded a $90 \%$ power at a 1 -sided type I error rate of $5 \%$ assuming a difference in median PFS between the arms of 2 months ( 2 months vs. 4 months), and assuming that 71 events would occur among 76 patients over the study period. An interim analysis was planned when half the required PFS events were observed (35). The trial would be stopped early for futility if the $P$ value from the log-rank statistic was greater than 0.5 (i.e., the PFS is worse for study Arm B than for study Arm A).

Descriptive statistics were used to summarize data for each individual biomarker at baseline and follow-up time points. The change at the follow-up time from baseline was calculated as the fold-change between the 2 time points. All those biomarker measurements were further compared between 2 treatment arms using Student's $t$ test. The association with clinical outcomes was explored using Cox proportional hazard model for time-to-event outcomes (e.g., OS or PFS) or logistic regression model for binary outcome (e.g., best response via RECIST). The biomarker value at baseline or its fold-change at a follow-up time point were further dichotomized by the median value noted above versus below-median for all available patients regardless of treatment group. The interaction effect between a dichotomized biomarker and treatment groups were tested in Cox and logistic regression model, in which we compared treatment arms (Arm B: cobimetinib + atezolizumab vs. Arm A: atezolizumab alone) inside each stratum by a biomarker. A significant interaction term indicates there is differential patient's response or outcome in treatments given their biomarker status. Such a biomarker is also referred to as a predictive biomarker. We used the 
same strategy for all available biomarkers collected, but only reported the ones that held significant interaction $P<0.05$.

Study approval. The study was approved by the NCI's Cancer Therapy Evaluation Program (CTEP) Institutional Review Board as well as the IRB or ethics committee at each participating institution, and was conducted in accordance with the provisions of the Declaration of Helsinki and the International Conference on Harmonization Guidelines for Good Clinical Practice. All patients provided written informed consent. The trial was registered under ClinicalTrials.gov as NCT03201458.

\section{Author contributions}

MY, NSA, and LC conceived and designed the study. MY, GBL, NSA and LC drafted the manuscript. LC, SG, and YL provided statistical analysis of primary and secondary outcomes. MY, NSA, and GBL obtained funding. MY, GBL, NSA, and LC supervised the study. MY, LC, ANR, RAA, AMN, LWG, LG, JL, DL, AKP, ARH, GKA, KS, EK, SLD, AJM, PRK, SG, YL, LD, SX, AAM, QZ, AWG, AP, HXC, ES, GBL, and NSA acquired, analyzed, or interpreted data. Additionally, all authors critically revised the manuscript for important intellectual content; provided administrative, technical, or material support; and reviewed, edited, and approved the manuscript. MY, NSA, and LC had access to all of the clinical data at all times, and the full data were shared with all of the study authors at the time the manuscript was submitted. The corresponding authors take responsibility for the integrity of the data and the accuracy of the data analysis.

\section{Acknowledgments}

We thank the patients and their families and all the investigators and site personnel. We acknowledge Teena Vithayathil for specimen biobanking; and Judy Murray, Jacob Titilola, and the Theradex team for technical and database support. This study was sponsored by NCI and conducted through the NCI's ETCTN. The study was also supported in part by NCI/NIH (R01 CA228414-01 and UM1CA186691); NCI's Specialized Program of Research Excellence (SPORE) in Gastrointestinal Cancers (P50 CA062924); NIH Center Core Grant (P30 CA006973); and the Passano Foundation. The study drugs used in this protocol were provided to the NCI under a Collaborative Agreement with Genentech/F. Hoffmann-La Roche Ltd. This paper was previously presented in part as an Oral Plenary at the American Association for Cancer Research (AACR) Annual Meeting, April 27-28, 2020 (Virtual Meeting), "A multicenter randomized phase 2 trial of atezolizumab as monotherapy or in combination with cobimetinib in biliary tract cancers (BTCs): A NCI Experimental Therapeutics Clinical Trials Network (ETCTN) study."

Address correspondence to: Nilofer Azad, 1650 Orleans Street, Room 4M10, Baltimore, Maryland 20815, USA. Phone: 410.955.8893; Email: nilo@jhmi.edu. Or to: Gregory B. Lesinski, Winship Cancer Institute of Emory University, 1365 Clifton Road NE, Atlanta, Georgia 30322, USA. Phone: 404.778.3072; Email: gregory.b.lesinski@emory.edu.
1. Patel T. Increasing incidence and mortality of primary intrahepatic cholangiocarcinoma in the United States. Hepatology. 2001;33(6):1353-1357.

2. Siegel RL, et al. Cancer statistics, 2016. CA Cancer JClin. 2016;66(1):7-30.

3. Khan SA, et al. Epidemiology, risk factors, and pathogenesis of cholangiocarcinoma. $H P B$ (Oxford). 2008;10(2):77-82.

4. Valle J, et al. Cisplatin plus gemcitabine versus gemcitabine for biliary tract cancer. $N$ Engl JMed. 2010;362(14):1273-1281.

5. Abou-Alfa GK, et al. Pemigatinib for previously treated, locally advanced or metastatic cholangiocarcinoma: a multicentre, open-label, phase 2 study. Lancet Oncol. 2020;21(5):671-684.

6. Abou-Alfa GK, et al. Ivosidenib in IDH1-mutant, chemotherapy-refractory cholangiocarcinoma (ClarIDHy): a multicentre, randomised, doubleblind, placebo-controlled, phase 3 study. Lancet Oncol.2020;21(6):796-807.

7. Lamarca A, et al. Second-line chemotherapy in advanced biliary cancer: a systematic review. Ann Oncol. 2014;25(12):2328-2338.

8. Rogers JE, et al. Second-line systemic treatment for advanced cholangiocarcinoma. J Gastrointest Oncol. 2014;5(6):408-413.

9. Lamarca A, et al. ABC-06|A randomised phase III, multi-centre, open-label study of active symptom control (ASC) alone or ASC with oxaliplatin / 5-FU chemotherapy (ASC+mFOLFOX) for patients (pts) with locally advanced / metastatic biliary tract cancers $(\mathrm{ABC})$ previously-treated with cisplatin/gemcitabine (CisGem) chemotherapy. JClin Oncol. 2019;37(15_suppl):4003-4003.

10. Lowery MA, et al. Second-line chemotherapy in advanced biliary cancers: A retrospective, multicenter analysis of outcomes. Cancer. 2019;125(24):4426-4434.

11. Schmitz KJ, et al. AKT and ERK1/2 signaling in intrahepatic cholangiocarcinoma. World J Gastroenterol. 2007;13(48):6470-6477.

12. Wang C, et al. A systems biology perspective on cholangiocellular carcinoma development: focus on MAPK-signaling and the extracellular environment. J Hepatol. 2009;50(6):1122-1131.

13. Yoon JH, et al. Enhanced epidermal growth factor receptor activation in human cholangiocarcinoma cells. J Hepatol. 2004;41(5):808-814.

14. Roberts PJ, Der CJ. Targeting the Raf-MEKERK mitogen-activated protein kinase cascade for the treatment of cancer. Oncogene. 2007;26(22):3291-3310.

15. Dudley DT, et al. A synthetic inhibitor of the mitogen-activated protein kinase cascade. Proc Natl Acad Sci U S A. 1995;92(17):7686-7689.

16. Finn RS, et al. A phase I study of MEK inhibitor MEK162 (ARRY-438162) in patients with biliary tract cancer. ASCO Meet Abstr. 2012;30(4_suppl):220.

17. Bekaii-Saab T, et al. Multi-institutional phase II study of selumetinib in patients with metastatic biliary cancers. J Clin Oncol. 2011;29(17):2357-2363.

18. Shroff RT, et al. The oral VEGF receptor tyrosine kinase inhibitor pazopanib in combination with the MEK inhibitor trametinib in advanced cholangiocarcinoma. Br J Cancer. 2017;116(11):1402-1407.

19. Lowery MA, et al. Binimetinib plus gemcitabine and cisplatin phase I/II trial in patients with advanced biliary cancers. Clin Cancer Res. 2019;25(3):937-945.
20. Kim RD, et al. A phase 2 multi-institutional study of nivolumab for patients with advanced refractory biliary tract cancer. JAMA Oncol. 2020;6(6):888-894.

21. Ueno M, et al. Pembrolizumab for advanced biliary adenocarcinoma: Results from the multicohort, phase II KEYNOTE-158 study. Ann Oncol. 2018;29(suppl_8):205-270.

22. Ioka T, et al. Evaluation of safety and tolerability of durvalumab (D) with or without tremelimum$\mathrm{ab}(\mathrm{T})$ in patients (pts) with biliary tract cancer (BTC). JClin Oncol. 2019;37(4_suppl):387-387.

23. Ueno M, et al. Nivolumab alone or in combination with cisplatin plus gemcitabine in Japanese patients with unresectable or recurrent biliary tract cancer: a non-randomised, multicentre, open-label, phase 1 study. Lancet Gastroenterol Hepatol. 2019;4(8):611-621.

24. Liu L, et al. The BRAF and MEK inhibitors dabrafenib and trametinib: effects on immune function and in combination with immunomodulatory antibodies targeting PD-1, PD-L1, and CTLA-4. Clin Cancer Res. 2015;21(7):1639-1651.

25. Dushyanthen S, et al. Agonist immunotherapy restores $\mathrm{T}$ cell function following MEK inhibition improving efficacy in breast cancer. Nat Commun. 2017;8(1):606.

26. Ebert PJR, et al. MAP kinase inhibition promotes $\mathrm{T}$ cell and anti-tumor activity in combination with PD-L1 checkpoint blockade. Immunity. 2016;44(3):609-621.

27. Loi S, et al. RAS/MAPK activation is associated with reduced tumor-infiltrating lymphocytes in triple-negative breast cancer: therapeutic cooperation between MEK and PD-1/PD-L1 


\section{CLINICAL MEDICINE}

immune checkpoint inhibitors. Clin Cancer Res. 2016;22(6):1499-1509.

28. Angell TE, et al. MHC class I loss is a frequent mechanism of immune escape in papillary thyroid cancer that is reversed by interferon and selumetinib treatment in vitro. Clin Cancer Res. 2014;20(23):6034-6044.

29. Mimura K, et al. The MAPK pathway is a predominant regulator of HLA-A expression in esophageal and gastric cancer. JImmunol. 2013;191(12):6261-6272.

30. Caunt CJ, et al. MEK1 and MEK2 inhibitors and cancer therapy: the long and winding road. Nat Rev Cancer. 2015;15(10):577-592.

31. Brea EJ, et al. Kinase regulation of human MHC class I molecule expression on cancer cells. Cancer Immunol Res. 2016;4(11):936-947.

32. Hellmann MD, et al. Phase Ib study of atezolizumab combined with cobimetinib in patients with solid tumors. Ann Oncol. 2019;30(7):1134-1142.

33. Eng C, et al. Atezolizumab with or without cobimetinib versus regorafenib in previously treated metastatic colorectal cancer (IMblaze370): a multicentre, open-label, phase 3, randomised, controlled trial. Lancet Oncol. 2019;20(6):849-861.

34. Dennison L, et al. Tumor and systemic immunomodulatory effects of MEK inhibition. Curr
Oncol Rep. 2021;23(2):23.

35. Yoo C, et al. Liposomal irinotecan (nal-IRI) in combination with fluorouracil (5-FU) and leucovorin (LV) for patients with metastatic biliary tract cancer (BTC) after progression on gemcitabine plus cisplatin (GemCis): Multicenter comparative randomized phase $2 \mathrm{~b}$ study (NIFTY). JClin Oncol.2021;39(15_suppl):4006-4006.

36. Javle $\mathrm{M}$, et al. Pertuzumab and trastuzumab for HER2-positive, metastatic biliary tract cancer (MyPathway): a multicentre, open-label, phase 2a, multiple basket study. Lancet Oncol. 2021;22(9):1290-1300.

37. Subbiah V, et al. Dabrafenib plus trametinib in patients with $\mathrm{BRAF}^{\mathrm{V} 600 \mathrm{E}}$-mutated biliary tract cancer (ROAR): a phase 2, open-label, singlearm, multicentre basket trial. Lancet Oncol. 2020;21(9):1234-1243.

38. Kim RD, et al. Randomised phase II trial (SWOG S1310) of single agent MEK inhibitor trametinib Versus 5-fluorouracil or capecitabine in refractory advanced biliary cancer. Eur J Cancer. 2020;130:219-227.

39. Plitas G, Rudensky AY. Regulatory T cells in cancer. Annu Rev Cancer Biol. 2020;4:459-477.

40. Tanaka A, Sakaguchi S. Targeting Treg cells in cancer immunotherapy. Eur J Immunol.
2019;49(8):1140-1146.

41. Saleh R, Elkord E. FoxP3 $3^{+}$T regulatory cells in cancer: prognostic biomarkers and therapeutic targets. Cancer Lett. 2020;490:174-185.

42. Dennison L, et al. Context-dependent immunomodulatory effects of MEK inhibition are enhanced with T-cell agonist therapy. Cancer Immunol Res. 2021;9(10):1187-1201.

43. Baumann D, et al. Proimmunogenic impact of MEK inhibition synergizes with agonist antiCD40 immunostimulatory antibodies in tumor therapy. Nat Commun. 2020;11(1):1-18.

44. Dushyanthen S, et al. Agonist immunotherapy restores $\mathrm{T}$ cell function following MEK inhibition improving efficacy in breast cancer. $\mathrm{Nat}$ Commun. 2017;8(1):606.

45. Lowery MA, et al. Comprehensive molecular profiling of intrahepatic and extrahepatic cholangiocarcinomas: Potential targets for intervention. Clin Cancer Res. 2018;24(17):4154-4161.

46. Yarchoan M, et al. Characterization of the immune microenvironment in hepatocellular carcinoma. Clin Cancer Res. 2017;23(23):7333-7339.

47. Farren MR, et al. Systemic immune activity predicts overall survival in treatment-naive patients with metastatic pancreatic cancer. Clin Cancer Res. 2016;22(10):2565-2574. 